\title{
Constrained Multi-objective Optimization Algorithm Based on $\varepsilon$ Adaptive Weighted Constraint Violation
}

\author{
Jue Wang ${ }^{1}$ \\ College of Information Science and Engineering, Dalian Polytechnic University \\ Dalian, 116034,China \\ E-mail: ragnarok18760163.com
}

Bo $\mathbf{L i}^{2}$

College of Information Science and Engineering,Dalian Polytechnic University

Dalian, 116034,China

E-mail: 1ibo_219vipe163.com

\section{Yinghong $\mathrm{CaO}^{3}$}

College of Information Science and Engineering,Dalian Polytechnic University

Dalian, 116034,China

E-mail: cao_yinghonge163.com

\begin{abstract}
A new constrained multi-objective optimization algorithm based on $\varepsilon$ adaptive weighted constraint violation, AW-CMOA, is proposed to solve the constrained multi-objective optimization problems. Considering different levels of difficulty in the satisfaction of varied constraints, a new $\varepsilon$ constraint handling strategy for multi-objective optimization, in which the constraint violation is redefined and the self-adapting $\varepsilon$ level parameter is set, is designed to make the constraint violation for individual to reflect its true quality more objectively and accurately. Simultaneously, according to the features of the constrained multi-objective and the evolutionary mechanism of $\mathrm{BBO}$, the model of constrained multi-objective optimization applicable to BBO is built. In the model, the habitat suitability index, in combination with the degree of feasible and the Pareto dominance relation between the individuals, is redefined. Moreover, the self-adaptive method of determining the migration rate is designed to improve the ability for exploitation and the utilization of better individual. Numerical experiments have shown that the proposed algorithm is competitive to other constrained multi-objective optimization algorithms in terms of convergence and distribution, and is capable of solving the complex constrained multi-objective optimization problems more effectively and efficiently.
\end{abstract}

CENet 2017

22-23 July, 2017

Shanghai, China

\footnotetext{
${ }^{1}$ Speaker

${ }^{2}$ This paper is supported by Doctoral Scientific Research Foundation of Liaoning Province of China (201501191,20160128), General Project for Scientific Research of Liaoning Province of China (L2015043), Youth Science Foundation of Dalian Polytechnic University (QNJJ201411)

${ }^{3}$ Correspongding Author
} 


\section{Introduction}

In the field of scientce, engineering and commerce, etc., many complex optimization problems involve multiple objectives and constraints, which are called constrained multiobjective optimization problems (CMOPs) [1]. Due to the conflicts of objectives, the interference among constraints, and the interrelationship between constraints and objectives, there are some difficulties in solving CMOPs [2]. Many optimization methods [3,4] have shown good search performance relatively, but there are still problems that it is easy to fall into the local optimal frontier and cannot take into account the convergence and distribution. Thus, the key of solving the problem lies in the effectiveness of constraint handling method $[5,6]$ and the efficiency of evolutionary mechanism. In addition, we also need to coordinate the balance between the constraint handling technology and the multi-objective evolutionary strategy. In order to obtain better solution performance on CMOPs, this paper proposes a new $\varepsilon$-constrained multi-objective handling mechanism. Moreover, On the basis of biogeography-based optimization (BBO)[7], a new constrained multi-objective optimization algorithm based on $\varepsilon$ adaptive weighted constraint violation (AW-CMOA) is proposed. The remainder of this paper is organized as follows. Section II presents a detailed description of AW-CMOA. Section III reports the simulation results and shows the performance of the proposed algorithm in comparison with other well-known optimization algorithms. Section IV presents the study conclusions.

\section{The proposed algorithm}

\subsection{Modified \&-constrained Handling Mechanism}

The main principle of $\varepsilon$-constraint handling technology is to measure the quality of the individual by comparing the level parameter $\varepsilon$ with the constraint violation degree of the individual. However, as the feasible space of CMOPs is often divided the constraint conditions into several isolated regions, at meanwhile, there is a certain degree of difference in satisfying different constraints. The degree of constraint violation is obtained by the linear summation may prompt the search to move fast toward the feasible region which is divided by constraint conditions satisfied easily. Thus some superior infeasible individuals with satisfying sectional constraints or a lower degree of constraint violation for the part but a little higher degree of constraint violation for the whole cannot be retained. Accordingly, there is a fault in the range of the individual. In the prophase of evolution, the algorithm may untimely fall into local optimum in the process of finding feasible region; therefore, in consideration of the difference of degree of difficulty satisfying various constraints, we need to carry out the research on the new determination method of constraint violation.

\subsubsection{Determination of Constraint Violation of iIdividuals}

In order to make the comprehensive constraint violation for individual to reflect the real degree of individual constraint violation more objectively, the adaptive $\varepsilon$ constraint violation degree determination method is proposed in Formula(2.1). A level parameter $\varepsilon_{j}$, is given for each constraint $j$, at meanwhile, the comparing results between the violation degree of each 
constraint $\varphi_{j}(x)$ and its level parameter $\varepsilon_{j}$, and the satisfaction degree of each constraint are added to the determination of the individual constraint violation degree.

$$
\phi(x)=\sum_{j=1}^{m} \frac{n_{j f e a}}{N P} \times\left(\max \left(0, \varphi_{j}(x)-\varepsilon_{j}(t)\right)\right)
$$

Where $N P$ is the total number of individuals in the current population; $n_{\text {fea }}$ is the number of individuals satisfying the $j$ th constraint $\left(\varphi_{j}(x)=0 \quad\right.$ ); $\varepsilon_{j}(t)$ is the level parameter of the $j$ th constraint at $t$ time, as determined by Formula (2.3) in the following Chapter. For CMOPs, in order to further measure the quality of individuals, we also need to integrate the Pareto dominance relationship of multi-objective into the comparisons among individuals. Therefore, in this paper, on the basis of the original $\varepsilon$ level comparison criterion, a new $\varepsilon$ constraint criterion is proposed by combining the Pareto dominance with the adaptive constraint violation degree $\phi(x)$. For individual $\boldsymbol{x}_{1}$ and $\boldsymbol{x}_{2}$ in population $\boldsymbol{H}$, the $\varepsilon$ constraint dominance relationship is shown as:

$$
x_{1}>_{\varepsilon} x_{2} \Leftrightarrow\left\{\begin{array}{c}
x_{1}>x_{2}, \phi\left(x_{i}\right)=\phi\left(x_{2}\right) \\
\phi\left(x_{i}\right)<\phi\left(x_{2}\right)
\end{array}\right.
$$

The new $\varepsilon$ constraint criterion evaluates the quality of the individual by substituting the comparison result of the parameter $\varepsilon$ and individual constraint violation degree into $\varepsilon$ constraint dominance relation.

\subsubsection{Determination Mechanism of Level Parameter $\varepsilon$}

In $\varepsilon$ constraint method, the comparison between level parameter $\varepsilon$ and the degree of individual constraint violation is substituted to the $\varepsilon$ constraint domination relation to reflect the quality of individual. However, the traditional method of determining the level parameter $\varepsilon$ has its own defects. The change trend of $\varepsilon$ only depends on the number of iterations and it does not take into account the quality of the individual. Thus, it is easy to cause the constraint pressure which the constraints of individuals are dominated by $\varepsilon$ smaller.

In the new constraint multi-objective handling mechanism proposed in this paper, the $\varepsilon$ constraint violation degree $\phi(x)$ of individuals can directly reflect the quality of individuals, but it is necessary to give a level parameter $\varepsilon_{j}$ for each constraint condition $j$. In view of the problem available in the original level parameter mentioned above, a new method is proposed here. For the $j$ th constraint, all of $\varepsilon_{j}$ of each generation is adaptively adjusted for the changes of feasible region according to the $j$ th constraint violation degree of the individual $x . \varphi_{j}(x)$ participates in adjusting the level parameters in the form of the proportion of it in the violation degree of the individual. The larger $\varphi_{j}(x)$ may affect the level parameter $\varepsilon_{j}$ with a smaller proportion, and the effect of the smaller $\varphi_{j}(x)$ on $\varepsilon_{j}$ may increase as its proportion increases. For each constraint condition, with the increase of the number of iterations, the determination of level parameters proposed in this paper can adaptively vary according to the individual constraint violation, and make full use of the effective information of the superior infeasible individuals, which can effectively improve the search efficiency of the feasible region. The determination of this new level parameter is shown as follows: 


$$
\varepsilon_{j}(t)=\left\{\begin{array}{c}
\frac{\sum_{i=1}^{N P} \varphi_{j}^{t}(x) \times \frac{\varphi_{j \max }^{t}-\varphi_{j}^{t}(x)}{\varphi_{j \max }^{t}-\varphi_{j \min }^{t}}}{\sum_{i=1}^{N P} \frac{\varphi_{j \max }^{t}-\varphi_{j}^{t}(x)}{\varphi_{j \max }^{t}-\varphi_{j \min }^{t}}}, \varphi_{j \max }^{t} \neq \varphi_{j \min }^{t} \\
0, \varphi_{\text {jmax }}^{t}=\varphi_{j \min }^{t}
\end{array}\right.
$$

Where $\varphi_{j}^{t}(x)$ is the constraint violation of individual $\boldsymbol{X}_{\boldsymbol{i}}$ at time $t ; \varphi_{\text {jmax }}^{t}$ and $\varphi_{\text {jmin }}^{t}$ are the maximum and minimum of $\varphi_{j}^{t}(x)$.

\subsection{Modified Determination Method of Habitat Suitability Index}

In view of the fact that the BBO algorithm itself is incapable of dealing with multiobjective optimization and the constraints, a multi-objective optimization model is designed in this paper, which is suitable to the BBO algorithm based on the new multi-objective constraint handling mechanism. At meanwhile, considering the greater effect of the performance of evolutionary strategy on the overall performance of the algorithm, in order to improve the constrained optimization ability of multi-objective optimization, the efficiency of BBO algorithm must be ensured. Therefore, it is necessary to redefine the Habitat suitability index (HSI) first. Since the evolution strategy of original BBO and its improved methods are only applicable to SOPs, it cannot meet the needs of multi-objective optimization, next, the evolution mechanism of the $\mathrm{BBO}$ is improved on the migration rate determination strategy.

In the BBO algorithm, the habitat suitability index (HSI) is an indicator of measuring the habitat quality. For CMOPs, in consideration of the characteristics of multi-objectives, it is necessary to determine HSI by combining Pareto dominance among habitat individuals. However, it is far from enough to define the HSI which is not enough to evaluate the quality of the habitat in this way and the constraints should also be taken into account fully. Thus it is ideal to design a determination mechanism of HSI, which can take into account the Pareto domination relationship and the degree of constraint satisfaction of individual itself. For this purpose, this paper proposes a new determination method of habitat suitability index HSI on the basis of the idea of $\varepsilon$ constraint domination mentioned above. First, the individuals in habitat population $\boldsymbol{H}=\left\{\boldsymbol{x}_{i}, i=1,2, \ldots, N P\right\}$ is ranked in ascending order in accordance with the adaptive $\varepsilon$ weighting constraint violation and form a new habitat population $\boldsymbol{H}$. Then, the $\varepsilon$ feasible habitat population $\boldsymbol{H}_{\text {zfea }}=\left\{\boldsymbol{x}_{i} \mid \phi\left(x_{i}\right)=0\right\}$ of current new population $\boldsymbol{H}$ is determined and the non-dominance degree $F_{i}$ of individual $\boldsymbol{x}_{\boldsymbol{i}}$ in $\boldsymbol{H}_{\varepsilon f e a}$ is calculated by Formula (2.4).

$$
F_{i}=\sum_{x_{i} \in H_{\varepsilon f f a}, x_{j}>x_{i}}\left\langle\frac{1}{N P} \mid\left\langle k \mid x_{j}, x_{k} \in H_{\varepsilon f e a} \wedge x_{j}>x_{k}, H_{\varepsilon f e a}=\left\langle x_{i} \mid \phi\left(x_{i}\right)=0\right\rangle\right\rangle\right\rangle
$$

Where $i, j, k \in\{1,2, \ldots, N P\} ; \quad x_{i}, x_{j}, x_{k} \in H \quad$ is the individual including $n$ dimensional suitability index variables. Finally, the HSI $G_{i}$ of the individual $\boldsymbol{x}_{\boldsymbol{i}}$ in the new habitat population $\boldsymbol{H}$ is determined as Formula (2.5).

$$
\begin{gathered}
G_{i}=\max \left(\phi\left(x_{i}\right), 0\right)+F_{i}^{\prime} \\
F_{i}^{\prime}=\left\{\begin{array}{c}
F_{i}, \phi\left(x_{i}\right)=0 \\
F_{\text {max }}, \phi\left(x_{i}\right) \neq 0
\end{array}\right.
\end{gathered}
$$




\subsection{Adaptive Determination Method of Migration Rate}

The habitat individual which needs to immigrate or emigrate in $\mathrm{BBO}$ algorithm is determined separately to carry out the species migration by migration rate. In order to reflect the quality of individuals in real time, an adaptive determination method of migration rate is proposed to ensure that it can make full use of the information of better individuals on different stages of evolution. The HSI of the normalized individuals is used to adaptively adjust the migration rate by combining the proportion of $\varepsilon$ feasible individuals in the current population and the proportion of non-dominated individuals in $\varepsilon$ feasible individuals. The adaptive immigration rate $\lambda$ and the emigration rate $\mu$ are determined in the following formula:

$$
\begin{gathered}
\lambda_{i}=\left(\frac{G_{i}-G_{\min }}{G_{\max }-G_{\min }}\right)^{N P^{1-(a+1)^{p}}} \\
\mu_{i}=1-\lambda_{i}
\end{gathered}
$$

Where $G_{i}$ is the HSI of $x_{i} ; G_{\max }$ and $G_{\min }$ are the maximum and minimum of HSI of current population; $a$ is the non-dominated rate of current $\varepsilon$ feasible population $H_{\varepsilon f e a}$, the proportion of non-dominated $\varepsilon$ feasible individuals in the number $n_{\varepsilon f e a}$ of $H_{\varepsilon f e a}$ individuals; $\quad \eta$ is the current $\varepsilon$ feasible rate, the proportion of $n_{\varepsilon f e a}$ in the total number $N P$ of habitat individuals. Under the premise of ensuring that the individual information of the current population can be effectively utilized, this method can reflect the individual's quality at real time, evaluate the immigration individual and emigration individual more accurately and scientifically.

\subsection{Description of AW-CMOA Algorithm}

Step 1. Initialize the parameter, which involves $m_{\max }, r_{\max }, r_{\min }, \beta$. Randomly generate NP individuals as initial population $H=\left\{x_{i}, i=1,2, \ldots, N P\right\}$.

Step 2. Calculate the objective function $F\left(x_{i}\right)=\left(f_{1}\left(x_{i}\right), \ldots, \mathrm{f}_{M}\left(x_{i}\right)\right)$ and the constraint violation degree $\gamma\left(x_{i}\right)=\left(\varphi_{1}\left(x_{i}\right), \ldots, \varphi_{m}\left(x_{i}\right)\right)$ of each habitat individual $x_{i}$.

Step 3.Calculate the $\varepsilon$ value under each constraint of $x_{i}$, and determine the $\varepsilon$ constraint violation degree $\phi\left(x_{i}\right)$ of $\mathrm{x}_{\mathrm{i}}$. Sort $\mathrm{x}_{\mathrm{i}}$ by its $\phi\left(x_{i}\right)$ in ascending order, reform a new population $H$. According to $\phi\left(x_{i}\right)$, determine the feasible population $\mathrm{H}_{\varepsilon f e a}$ in $H$, calculate the non-dominated degree $F_{i}$ of individal in $\mathrm{H}_{\varepsilon f e a}$, thus obtain the HSI value $G_{i}$ of each habitat individual $x_{i}$.

Step 4. Sort the habitat individuals of $H$ by HSI in ascending order, reserve the $N P$ individuals with better distribution and non-dominated as current optimal habitat population $H$.

Step 5. Determine the species immigration rate $\lambda_{i}$ and the emigration rate $\mu_{i}$ of $x_{i}$ in $H$, and calculate the species probability $P_{i}$ and mutation rate $m_{i}$. On the basis of immigration rate $\lambda_{i}$ and the emigration rate $\mu_{i}$, make migration and mutation, and thus obtain new population $H$. Judge whether it satisfies the termination condition or not. If it satisfies the condition, go to Step 6 ; otherwise go to Step 2.

Step 6. Output the non-dominated feasible population $H$.

\section{Simulation}

In this section, in order to evaluate the performance of AW-CMOA on solving the constrained multi-objective optimization problem, it is compared with several constrained 
multi-objective optimization algorithms which are the most representative and best optimized such as C-PSA[2], MOABC[3] and CMOPSO[4] on the accepted test functions TNK, SRN, CONSTR and OSY[4]. The initial population size of AW-CMOA and the other 3 algorithms are set as 100. In AW-CMOA, $\mathrm{m}_{\max }=0.005, \mathrm{r}_{\max }=0.9, \mathrm{r}_{\min }=0.4, \beta=0.02$. Furthermore, the other parameters are set as described in the comparison algorithm. Three evaluation criteria [8] as two set coverage (C), generational distance metric (GD) and space metric (SP) are used together to test the performance of each algorithm. For fair comparison, each algorithm is run independently 40 times and the algorithm stops when the number of function evaluation per run reaches 25000 .

Table 1 shows the experimental results of 4 algorithms for the 4 standard test functions on the $\mathrm{C}$ index including the average (outside brackets) and standard deviation (in brackets). The mutual dominance relation between the two sets of solutions is reflected directly to measure the relative convergence of the solution set. The statistical box figure of $\mathrm{C}$ index among AW-CMOA and other 3 algorithms is shown in Fig. 1, where AW-CMOA, C-PSA, MOABC and CMOPSO is expressed as $1 \sim 4$. For 4 test functions, $C(1,2)$ is greater than $C(2,1)$. It shows that the solution obtained by the proposed algorithm AW-CMOA features a higher degree of dominance over the solution obtained by C-PSA. Similarly, the other results are $C(1,3)$ greater than $C(3,1)$, $\mathrm{C}(1,4)$ greater than $\mathrm{C}(4,1)$. It shows that the proportion of the solution which can be dominated by the solution set of AW-CMOA in C-PSA, MOABC and CMOPSO is much larger than the proportion of the solution that can dominate the solution of the AW-CMOA. Therefore, the convergence of the Pareto optimal front of AW-CMOA is better than that of other 3 algorithms.

\begin{tabular}{ccc|cc|cc}
\hline Test Function & $\mathrm{C}(1,2)$ & $\mathrm{C}(2,1)$ & $\mathrm{C}(1,3)$ & $\mathrm{C}(3,1)$ & $\mathrm{C}(1,4)$ & $\mathrm{C}(4,1)$ \\
\hline TNK & $0.48(0.00432)$ & $0.07(0.00212)$ & $0.65(0.00738)$ & $0.01(0.00026)$ & $0.55(0.00219)$ & $0.11(0.00038)$ \\
SRN & $0.59(0.00763)$ & $0.19(0.00216)$ & $0.75(0.00335)$ & $0.14(0.00127)$ & $0.54(0.00094)$ & $0.21(0.00081)$ \\
CONSTR & $0.47(0.00375)$ & $0.04(0.00073)$ & $0.61(0.00742)$ & $0.02(0.00033)$ & $0.38(0.00988)$ & $0.03(0.00050)$ \\
OSY & $0.46(0.00532)$ & $0.02(0.00047)$ & $0.85(0.00453)$ & $0.03(0.00112)$ & $0.63(0.00136)$ & $0.01(0.00035)$ \\
\hline
\end{tabular}

Table 1: Statistical Results of $C$ indicator by 4 Algorithms on 4 Functions

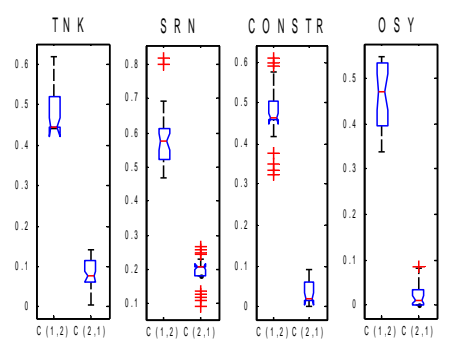

(a) $\mathrm{C}(1,2)$ and $\mathrm{C}(2,1)$

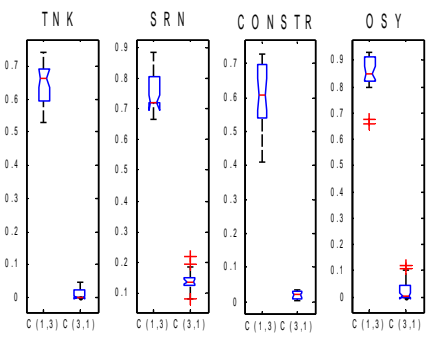

(b) $\mathrm{C}(1,3)$ and $\mathrm{C}(3,1)$

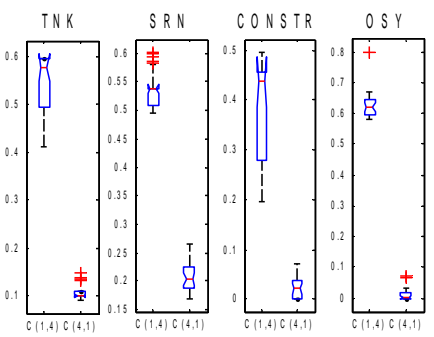

(c) $\mathrm{C}(1,4)$ and $\mathrm{C}(4,1)$

Figure 1:Statistical Box of C Indicator by 4 Algorithms

Considering that the comparison results of $\mathrm{C}$ index is largely dependent on the contrast algorithm, Table 2 shows the experimental results for the 4 test functions on the GD index for the purpose of further analyzing the convergence performance of the 4 algorithms. The statistical box figure of GD index is shown in Fig. 2. As seen from Table 2 and Fig. 2, for 4 test functions, compared with C-PSA, MOABC and CMOPSO, AW-CMOA can not only obtain the minimum average GD value, but also the minimum GD value which is less than the minimum value obtained by other algorithms. It is shown that the results obtained by AW-CMOA are 
closer to the ideal Pareto front and have obvious advantages in convergence. At the same time, as seen from Fig. 2, the outliers in the data obtained by AW-CMOA are less than other algorithms, which indicates that AW-CMOA has the best convergence stability in the 4 algorithms.

\begin{tabular}{ccccccccc}
\hline Test Function & \multicolumn{2}{c}{ CMOBBO } & \multicolumn{2}{c}{ C-PSA } & \multicolumn{2}{c}{ MOABC } & \multicolumn{2}{c}{ CMOPSO } \\
\hline TNK & $9.23 \mathrm{E}-05$ & $(3.88 \mathrm{E}-07)$ & $4.17 \mathrm{E}-04$ & $(1.86 \mathrm{E}-05)$ & $7.11 \mathrm{E}-04$ & $(2.04 \mathrm{E}-05)$ & $7.82 \mathrm{E}-04$ & $(7.72 \mathrm{E}-05)$ \\
SRN & $3.83 \mathrm{E}-04$ & $(5.01 \mathrm{E}-06)$ & $6.29 \mathrm{E}-04$ & $(8.47 \mathrm{E}-06)$ & $2.75 \mathrm{E}-03$ & $(3.98 \mathrm{E}-04)$ & $9.12 \mathrm{E}-04$ & $(3.77 \mathrm{E}-05)$ \\
CONSTR & $6.74 \mathrm{E}-05$ & $(5.32 \mathrm{E}-06)$ & $8.98 \mathrm{E}-05$ & $(3.08 \mathrm{E}-06)$ & $7.12 \mathrm{E}-04$ & $(1.06 \mathrm{E}-05)$ & $4.84 \mathrm{E}-04$ & $(2.75 \mathrm{E}-05)$ \\
OSY & $6.06 \mathrm{E}-04$ & $(7.25 \mathrm{E}-06)$ & $5.14 \mathrm{E}-03$ & $(1.40 \mathrm{E}-05)$ & $1.38 \mathrm{E}-02$ & $(3.41 \mathrm{E}-03)$ & $7.73 \mathrm{E}-03$ & $(5.12 \mathrm{E}-05)$
\end{tabular}

Table 2: Statistical Results of GD Indicator by 4 Algorithms on 4 Functions
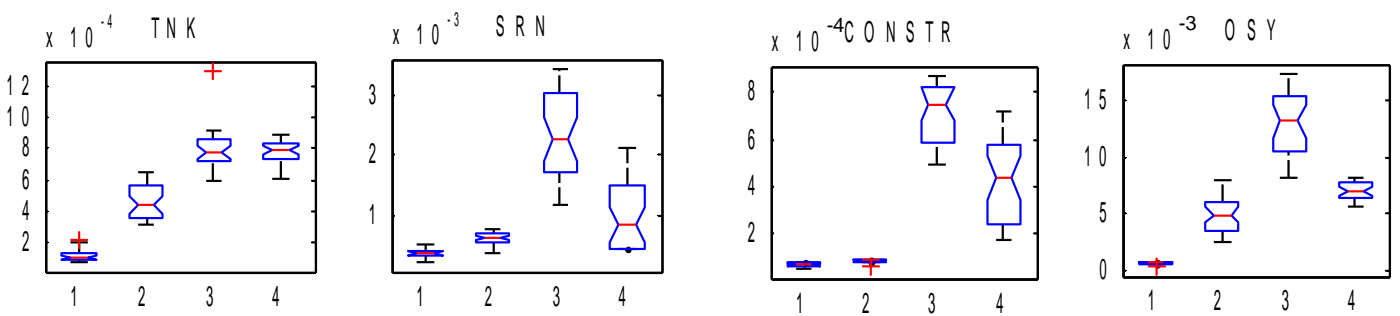

Figure 2: Statistical Results of GD Indicator by 4 Algorithms on 4 Functions

For the purpose of evaluating the distribution of the solution set of 4 algorithms, Table 3 shows the experimental results on the SP index, the SP index of each algorithm is shown in Fig. 3. It is not difficult to see from Table 3 and Fig. 3, for 4 test functions, the SP value obtained by AW-CMOA is the least and the outliers in the statistical data are the least, which indicates that AW-CMOA has more advantages than the other 3 algorithms in distribution, the approximate Pareto optimal solution set can be distributed more uniformly.

\begin{tabular}{ccccccccc}
\hline Test function & \multicolumn{2}{c}{ CMOBBO } & \multicolumn{2}{c}{ C-PSA } & \multicolumn{2}{c}{ MOABC } & \multicolumn{2}{c}{ CMOPSO } \\
\hline TNK & $7.12 \mathrm{E}-03$ & $(2.23 \mathrm{E}-05)$ & $8.82 \mathrm{E}-03$ & $(7.03 \mathrm{E}-05)$ & $1.91 \mathrm{E}-02$ & $(4.46 \mathrm{E}-03)$ & $1.89 \mathrm{E}-02$ & $(3.33 \mathrm{E}-03)$ \\
SRN & $5.86 \mathrm{E}-03$ & $(8.20 \mathrm{E}-05)$ & $1.15 \mathrm{E}-02$ & $(8.22 \mathrm{E}-03)$ & $3.93 \mathrm{E}-02$ & $(6.24 \mathrm{E}-03)$ & $2.85 \mathrm{E}-02$ & $(5.86 \mathrm{E}-03)$ \\
CONSTR & $4.48 \mathrm{E}-02$ & $(3.93 \mathrm{E}-04)$ & $7.41 \mathrm{E}-02$ & $(9.12 \mathrm{E}-04)$ & $9.01 \mathrm{E}-02$ & $(6.15 \mathrm{E}-03)$ & $8.79 \mathrm{E}-02$ & $(9.12 \mathrm{E}-04)$ \\
OSY & $1.12 \mathrm{E}-01$ & $(8.84 \mathrm{E}-04)$ & $6.17 \mathrm{E}-01$ & $(9.23 \mathrm{E}-03)$ & $8.67 \mathrm{E}-01$ & $(1.48 \mathrm{E}-02)$ & $7.28 \mathrm{E}-01$ & $(8.63 \mathrm{E}-03)$ \\
\hline
\end{tabular}

Table 3: Statistical Results of SP Indicator by 4 Algorithms on 4 Functions
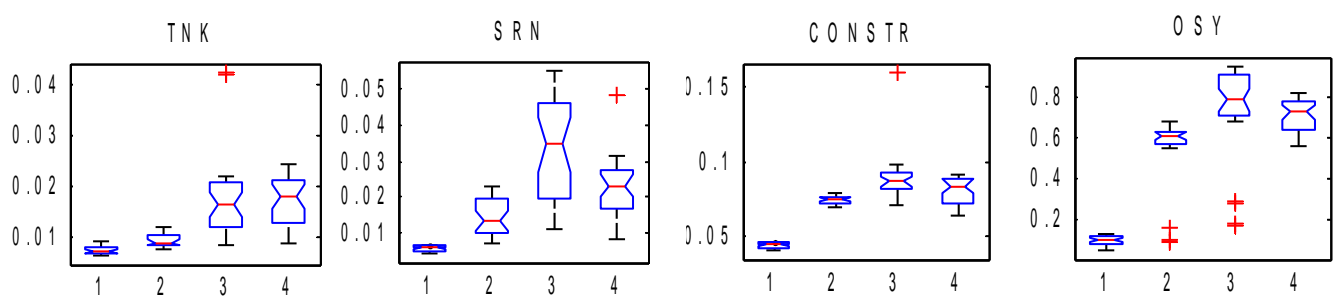

Figure 3: Statistical Results of SP Indicator by 4 Algorithms on 4 Functions

Based on the above analysis, it is found that both the convergence and the distribution uniformity, AW-CMOA has obvious advantages compared with the other 3 algorithms, and can obtain an approximate Pareto optimal solution set which is closer to the ideal Pareto front and more uniform distribution. 


\section{Conclusion}

In this paper, the constrained multi-objective optimization algorithm based on $\varepsilon$ adaptive weighted constraint violation (AW-CMOA) is proposed. It shows a $\varepsilon$ multi-objective constraint handling method by the following mechanism. On one side, taking into account the difficulty difference in the satisfaction of the different constraints, an adaptive weighted determination method of $\varepsilon$ constraint violation is proposed to reflect the constraint satisfaction degree of individuals more objectively. On the other side, according to the violation degree of each constraint of the individual, the level parameters are adaptively adjusted to enhance the ability of search purposively for feasible region. After determining the constraint handling method, in combination with the multi-objective constraint handling method mentioned above and the mechanism of BBO itself, a constrained multi-objective optimization model suitable for BBO is established. In this model, the habitat suitability index is redefined. After that, on the basis of preserving the original idea of $\mathrm{BBO}$, the new evolutionary mechanism for AW-CMOA is redesigned. The simulation results show that the AW-CMOA proposed in this paper can improve the convergence and distribution uniformity of the solution sets by comparing with other algorithms, and can effectively and efficiently solve the multi-objective optimization problem of complex constraints.

\section{References}

[1] F, Zhun. Angle-based constrained dominance principle in MOEA/D for constrained multi-objective optimization problems[C].IEEE Congress on Evolutionary Computation IEEE, 2016:460-467

[2] H.K.Singh,T.R.,Smith Warren.C-PSA: Constrained Pareto simulated annealing for constrained multi-objective optimization[J]. Information Sciences, 2010, 180(13):2499-2513

[3] R.Akbari,R.Hedayatzadeh,K.Ziarati,st al.A multi-objective artificial bee colony algorithm[J].Swarm and Evolutionary Computation, 2012 ,2(2):39-52

[4] G.G.Yen, W.F.Leong.Constraint handling procedure for multiobjective particle swarm optimization[C].IEEE World Congress on Computational Intelligence, WCCI 2010 - 2010 IEEE Congress on Evolutionary Computation, CEC 2010,2010:503-521

[5] D, Rituparna, R. G. Regis. A surrogate-assisted evolution strategy for constrained multi-objective optimization[J]. Expert Systems with Applications,2016,57(2):270-284

[6] M, Minami, K. Takadama, H. Sato. Controlling selection areas of useful infeasible solutions for directed mating in evolutionary constrained multi-objective optimization [J]. Annals of Mathematics and Artificial Intelligence,2016,76(1):25-46

[7] D.Simon. Biogeography-based optimization [J].IEEE Transactions on Evolutionary Computation, 2008, 12(6):702-713.

[8] L.Tang, X.P.Wang. A hybrid multi- objective evolutionary algorithm for multi-objective optimization problems [J].IEEE Transactions on Evolutionary Computation, 2013, 17(1):20-45 